\title{
REDUCTIONS OF IDEALS IN PRÜFER DOMAINS
}

\author{
JAMES H. HAYS
}

\begin{abstract}
All rings under consideration are Prüfer domains or valuation domains. We characterize the set of basic ideals and the set of $C$ ideals in an arbitrary valuation ring. Basic ideals were introduced in 1954 by Northcott and Rees. The concept of a $C$-ideal is, in a sense, directly opposite to that of a basic ideal. We then prove that a necessary and sufficient condition for every ideal in a domain $D$ to be basic is that $D$ be a one-dimensional Prüfer domain.
\end{abstract}

Introduction. An ideal $B$ is a reduction of the ideal $A$ if $B \subseteq A$ and $B A^{n}=A^{n+1}$ for some positive integer $n$. An ideal is basic if it has no proper reductions and is a $C$-ideal if it is not a reduction of any larger ideal.

The first two of the above definitions appeared originally in a paper by Northcott and Rees [3]. The setting of that paper was a local ring with an infinite residue field. They established a nice description of basic ideals in those rings: an ideal is basic if and only if it can be generated by analytically independent elements. It was also shown that an ideal $B$ which does not consist entirely of zero divisors is a reduction of the ideal $A$ if and only if the elements of $A$ are analytically dependent on $B$. This condition was also shown to he equivalent to the existence of an ideal $C$ with $B C=A C$. These last two results would thus describe $C$-ideals in that setting.

In [2], we investigated reductions of ideals in arbitrary commutative rings. In Noetherian rings, basic ideals were characterized to the extent that they are characterized in local rings. It was also shown that elements of the principal class, a generalization of analytic independence, generate basic ideals but not conversely. The basic properties of $C$-ideals were also examined.

We also showed that a domain is Prüfer if and only if each finitely generated ideal is basic [2, Theorem 6.5, p. 62]. It was also shown that in a one-dimensional Prüfer domain, every ideal is basic; that is, the basic ideal property holds [2, Theorem 6.1, p. 61]. We also observed that the basic ideal property need not hold for all Prüfer domains.

These facts suggest that in a Prüfer domain of dimension greater than one, there are ideals which are not basic. We establish this in Theorem 10.

Received by the editors March 11, 1974 and, in revised form, August 12, 1974. AMS (MOS) subject classifications (1970). Primary 13A15, 13F05; Secondary $13 \mathrm{C} 05$.

Key words and phrases. Reductions of ideals, basic, $C$-ideals, Prüfer domains, valuation rings, primary ideals, one-dimensional. 
In particular, we prove that a domain $D$ has the basic ideal property if and only if $D$ is a one-dimensional Prüfer domain. Theorem 3 characterizes basic ideals in valuation rings. We also prove that in a valuation ring, an ideal is a $C$-ideal if and only if it is primary [Theorem 7].

In the proof of $[2$, Theorem 6.1, p. 61], we establish the following result which we list here for convenience.

Lemma A. Let $(V, M)$ be a valuation ring. An ideal $A$ with $\operatorname{rad} A=M$ is basic.

Proper inclusion is denoted by " $C$ ". All ideals under consideration are proper ideals and if $P$ is a prime ideal in the domain $D$, then $e_{P}$ and $c_{P}$ denote extension and contraction with respect to the quotient ring $D_{P}$.

2. Results. We first observe that in a Prüfer domain the definition of a reduction can be restricted.

Proposition 1. If $B$ is a reduction of the ideal $A$ in Prüfer domain $D$, then $B A=A^{2}$.

Proof. Let $B A^{n}=A^{n+1}$, then $3^{n} A^{n}=A^{2 n}$. This equation shows that $(B A)^{e}=\left(A^{2}\right)^{e}$ in each $D_{M}$, where $M$ is a maximal ideal of $D$; thus the result follows from [1, Theorem 3.10 , p. 42].

The following lemma follows easily from Proposition 1.

Lemma 2. An ideal $A$ in a valuation ring has a principal reduction $(x)$ if and only if $(x) \subseteq A$ and $\left[A^{2}:(x)\right]=A$.

A characterization of basic ideals in a valuation ring is now available. The proof is easy and is omitted.

Theorem 3. A nonzero ideal in a valuation ring is basic if and only if one of the following holds:

(1) $A$ is principal.

(2) $A=A^{2}$.

(3) $A \subset\left[A^{2}:(x)\right]$ for each $x \in A$.

The next result is an important tool in the remainder of the paper.

Lemma 4. Let $x$ be a nonzero element of the valuation ring $V$ and let $\operatorname{rad}(x)=P$. Then $(x)$ is a reduction of $(x)^{e} P^{c} P$.

Proof. Let $Q=(x)^{e} P^{c} P$ and note that $Q^{2} \subset(x) \subseteq Q$. Let $a \in\left[Q^{2}:(x)\right]$, then there exists an $s$ not in $P$ such that $s a x \in\left(x^{2}\right)$. This implies that $a \in Q$ and thus $(x)$ is a reduction of $Q$ by Lemma 2 .

We have previously characterized the basic ideals in a valuation domain. By making use of Lemma 4 , we are able to do the same for $C$-ideals. 
Theorem 5. An ideal $A$ in a valuation ring is a $C$-ideal if and only if $A$ is a primary ideal.

Proof. Let $A$ be a $C$-ideal with $\operatorname{rad} A=P$ and let $A^{e} P^{c} P=Q$. If $A=$ $Q$, then $A$ is primary; so assume $A \subset(x) \subseteq Q$. We observe that $(x)^{e} P^{c} P=Q$. It then follows from Lemma 4 and Proposition 1 that $(x) Q=Q^{2}$. By the definition of $Q$, there exists an element $s \notin P$ such that $(s) Q^{2}=(s)(x) Q \subseteq$ $A Q$. However, $(s) Q^{2}=Q^{2}$ by $[1$, Theorem 14.3, p. 173] and thus $A$ is a reduction of $Q$. Hence $A$ is primary.

The converse follows easily from Lemma $A$ applied in $D_{P}$.

In fact the converse of the preceding depended only on $D_{P}$ being a valuation ring and thus the following result was verified.

Corollary 6. A primary ideal in a Prüfer domain is a C-ideal.

Proposition 7. Let $A$ be a nonidempotent ideal in a valuation ring with $\operatorname{rad} A=P$, a nonmaximal prime ideal. The following are equivalent:

(1) $A$ is a basic primary ideal.

(2) $A^{e} P$ is not principal.

(3) $A$ is the union of $P$-primary ideals $\left\{u_{a}\right\}, u_{a} \neq A$.

Proof. (1) $\Rightarrow(2)$ Lemma 4 insures that a basic primary ideal does not have a principal extension. The implications $(2) \Rightarrow(3)$ and $(3) \Rightarrow(1)$ are straightforward.

Theorem 8. Let $A$ be an ideal in a Prüfer domain with a finite number of minimal prime ideals; then $A$ is a $C$-ideal if and only if $A$ is the intersection of primary ideals.

Proof. Let $P_{1}, \ldots, P_{n}$ be the minimal prime ideals of the $C$-ideal $A$ and let $Q_{i}$ be the isolated $P_{i}$ components of $A$. We will show that $A$ is a reduction of $Q=\bigcap_{i=1}^{n} Q_{i}$ and thus that $A=Q$. It suffices to show that $A^{e} M Q^{e} M=\left(Q^{2}\right)^{e} M$ for each maximal ideal $M$. Since a maximal ideal $M$ contains only one $P_{i}$, this follows immediately from the proof of Theorem 5 .

Conversely, if $A$ is an intersection of primary ideals then the fact that $A$ is a $C$-ideal follows from [2, Theorem 3.2, p. 59] and Corollary 6.

We note the preceding theorem extends Theorem 5 to ideals in Prüfer domains with prime radical.

Proposition 9. Let $x$ be a nonzero element of a Prüfer domain $D$ and let $P$ be a minimal prime of $B$. Let $(x)^{e} P^{c} P=B$.

(1) $\left[(x)+B^{2}\right] B=B^{2}$.

(2) If $B$ is basic, then $P$ is a maximal ideal.

Proof. To prove (1), it is sufficient to show that $\left[(x)+B^{2}\right]^{e} M_{B}{ }^{e} M=$ $\left(B^{2}\right)^{e} M$ for each maximal ideal $M$ that contains $B$. We note that $B^{e} M=$ 
$\left\{r \in D_{M} \mid r s \in(x)^{e} M\right.$ for some $\left.s \notin P^{e} M\right\}$ and that $\left[(x)+B^{2}\right]^{e} M=(x)^{e} M$. The result then follows from Lemma $4 .^{1}$

To prove (2) let $B$ be basic; then $(x)+B^{2}=B$. Let $y$ be any element not in $P$. It is easy to see that $P$ is a minimal prime of $(y x)$ and that $(y x)^{e} P^{c} P=B$. By (1), we observe that $x \in(y x)+B^{2}$. This implies that $x-y x r=x(1-y r) \in B^{2}$ for some $r \in R$. Hence $x(1-y r) s \in(x)^{2}$ for some $s \notin P$ and therefore $1-y r \in P$. Thus $P$ is maximal.

We observe that Proposition 9 applies to all nonidempotent prime ideals of a Prüfer domain. It also provides the following characterization of the basic ideal property.

Theorem 10. An integral domain has the basic ideal property if and only if it is a one-dimensional Prüfer domain.

Proof. That a one-dimensional Prüfer domain has the basic ideal property was established in [2, Theorem 6.1, p. 61].

Now let $D$ be an integral domain in which every ideal is basic. We know that $D$ must be Prüfer [2, Theorem 6.5, p. 62], so we only need show that $D$ has dimension one. Suppose not, then there exist prime ideals $P$ and $M$ such that $(0) \subset P \subset M$. Let $x$ be a nonzero element of $P$ and let $Q$ be the minimal prime of $(x)$ contained in $P$. Then the $Q$-primary component of $(x)$ is not basic by Proposition 9, a contradiction.

\section{BIBLIOGRAPHY}

1. R. Gilmer, Multiplicative ideal theory, Queen's Papers in Pure and Appl. Math., no. 12, Queen's University, Kingston, Ontario, 1968. MR 37 \#5198.

2. J. Hays, Reductions of ideals in commutative rings, Trans. Amer. Math. Soc. 177 (1973), 51-63.

3. D. G. Northcott and D. Rees, Reductions of ideals in local rings, Proc. Cambridge Philos. Soc. 50 (1954), 145-158. MR 15, 596.

4. O. Zariski and P. Samuel, Commutative algebra. Vol. 1, University Ser. in Higher Math., Van Nostrand, Princeton, N. J., 1958. MR 19, 833.

DEPARTMENT OF MATHEMATICS, UNIVERSITY OF NEW ORLEANS, NEW ORLEANS, LOUISIANA 70122

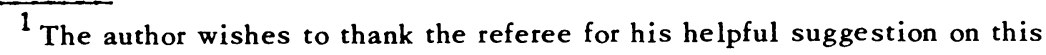
argument. 